\title{
Predictive value of blood pressure variability for organ dysfunction in patients with sepsis
}

\author{
Yiyun Zhu, Yunfei Li, Weiwei Cai, Chunmei Zhang \\ Department of Critical Medicine, The Second Affiliated Hospital and Yuying Children's Hospital of Wenzhou Medical University, Wenzhou, China \\ Contributions: (I) Conception and design: Y Zhu; (II) Administrative support: C Zhang; (III) Provision of study materials or patients: All authors; \\ (IV) Collection and assembly of data: All authors; (V) Data analysis and interpretation: All authors; (VI) Manuscript writing: All authors; (VII) Final \\ approval of manuscript: All authors. \\ Correspondence to: Chunmei Zhang. Chief nurse, The Second Affiliated Hospital and Yuying Children's Hospital of Wenzhou Medical University, 109 \\ Xueyuan West Road, Lucheng District, Wenzhou, China. Email: sallyzcm@126.com.
}

\begin{abstract}
Background Sepsis is a common disease in the intensive care unit, accompanied by many complications and high mortality. Organ dysfunction is a major risk factor for death in patients with sepsis. Analyzing the related factors of organ dysfunction caused by sepsis can provide more clinical prevention and treatment targets, and better predict patients' prognoses. This study aimed to investigate the relationship between blood pressure variability (BPV) and organ dysfunction in patients with sepsis.
\end{abstract}

Methods: One hundred and two patients with sepsis treated in our hospital from January 2019 to January 2021 were retrospectively collected as an observation group, and 102 healthy people were collected as a control group. The 24-hour systolic blood pressure variability (24h SBPV) and 24-hour diastolic blood pressure variability (24h DBPV) of the two groups were compared, and the correlation between 24h SBPV, $24 \mathrm{~h}$ DBPV, and organ function damage to sepsis patients were analyzed.

Results: Compared with the control group, the $24 \mathrm{~h}$ DBPV in the observation group was significantly higher $(0.56 \pm 0.16$ vs. $0.37 \pm 0.16, \mathrm{P}=0.000)$. 24h SBPV increased significantly $(0.56 \pm 0.16$ vs. $0.36 \pm 0.17$, $\mathrm{P}=0.000)$. Pearson linear correlation analysis showed $24 \mathrm{~h}$ SBPV was positively correlated with the level of procalcitonin, APACHEII score, and SOFA score $(\mathrm{r}=0.301,0.216$ and $0.218, \mathrm{P}<0.05)$. 24h DBPV was positively correlated with the level of procalcitonin, APACHEII score, and SOFA score ( $\mathrm{r}=0.302,0.212$ and $0.210, \mathrm{P}<0.05)$. 24h SBPV and $24 \mathrm{~h}$ DBPV are of certain value in the diagnosis of multiple organ failure in patients with sepsis, and the area under the curve was [0.649 (95\% CI: 0.539-0.759), P=0.010] and [0.650 (0.540-0.760), $\mathrm{P}=0.009$ ], respectively. 24h SBPV and 24h DBPV are of certain value in the diagnosis of persistent organ failure in patients with sepsis, and the area under the curve was [0.647 (95\% CI: 0.538-0.757), $\mathrm{P}=0.010]$ and [0.647 (95\% CI: 0.538-0.757), $\mathrm{P}=0.010]$, respectively.

Conclusions: The increase of BPV in patients with sepsis has a certain value in predicting the prognosis and organ function damage to patients with sepsis.

Keywords: Blood pressure variability (BPV); sepsis; organ function; prediction

Submitted Jul 05, 2021. Accepted for publication Sep 01, 2021.

doi: $10.21037 / \mathrm{apm}-21-2183$

View this article at: https://dx.doi.org/10.21037/apm-21-2183

\section{Introduction}

Sepsis is an organ dysfunction syndrome caused by the imbalance of the human response to infection. It is mainly characterized by chills, fever (or hypothermia), palpitation, shortness of breath, mental state changes, etc. Sepsis can develop into severe sepsis and septic shock, leading to organ dysfunction, circulatory disorder, and high mortality. Worldwide, the number of patients with sepsis can reach tens of millions yearly. The mortality is more than $25 \%$, 
and some patients may have different degrees of organ dysfunction after survival. Severe organ dysfunction caused by sepsis is a significant cause of death. Analyzing the factors related to sepsis organ function injury can provide more clinical prevention and sepsis-related organ function injury targets and better predict patients' prognosis. Studies have shown that blood pressure variability (BPV) is related to organ function injury in various diseases, but relevant studies focus mainly on patients with cardiovascular diseases (1-3). The common pathophysiological basis of functional injury of related organs caused by sepsis is that patients cannot maintain blood pressure and withstand the attack of many inflammatory factors. The disorder of the autonomic nervous system plays a vital role in this process. The change of BPV has been proved to be related to autonomic nervous dysfunction. Therefore, BPV may play an essential role in sepsis-related organ dysfunction. In 2017, the research conducted by Chinese scholars showed that the change of systolic blood pressure variability (SBPV) was related to the 28-day mortality of patients (4). A foreign study in 2018 showed that the change of BPV was related to the increase of blood lactate concentration and SOFA score (5). These two studies suggest that BPV is related to the severity of sepsis, but the number of cases in these two studies is small, and the relationship between BPV and functional damage to different organs is not deeply discussed. The primary purpose of this study was to explore the correlation between BPV and target organ function injury in patients with sepsis. We present the following article in accordance with the STARD reporting checklist (available at https://dx.doi. org/10.21037/apm-21-2183).

\section{Methods}

\section{General data}

One hundred and two patients with sepsis treated in our hospital from January 2019 to January 2021 were retrospectively collected as the observation group. The inclusion criteria are as follows: (I) patients met the Sepsis 3.0 Diagnostic Criteria (6); (II) aged 18-65; (III) BPV data can be obtained. Exclusion criteria: (I) previous insufficiency of liver, kidney, heart, brain, or lung; (II) immune system diseases including ulcerative colitis; (III) pregnant women; (IV) complicated with malignant tumor; (V) blood system diseases; (VI) hypertension, diabetes and so on; (VII) undergone organ transplantation or long-term use of immunosuppressants; (VIII) other serious diseases, such as hemophilia and dementia. In the same period, 102 healthy people were collected as the control group. None of the healthy people had the above diseases or other diseases. This study is a retrospective clinical study that can do without patient informed consent. The protocol of this study is in line with the Declaration of Helsinki (as revised in 2013). It retrospectively analyzes the imaging and clinical data left by patients, and does not use serum or tissue for additional tests. It is an observational test that can do without the approval of the ethics committee.

\section{Treatment strategies}

According to the international guidelines for sepsis treatment (6), all patients were given symptomatic and supportive treatments, such as early anti-infection, control of primary diseases, intensive care, fluid resuscitation, maintenance of water-electrolyte and acid-base balance, and organ functional support, such as continuous hemofiltration and mechanical ventilation when necessary.

\section{Observed indicators}

Main outcome measures include the level of procalcitonin, the number of leukocytes, acute physiology and chronic health evaluation (APACHE II), sequential organ failure score (SOFA), multiple organ function injury, persistent organ function injury, cardiac insufficiency, acute renal injury, acute respiratory distress syndrome, continuous renal replacement therapy, mechanical ventilation, and 28-day mortality.

\section{Definitions}

(I) Organ failure: if an organ scores two or more points in each score of SOFA, it is diagnosed as organ failure. Multiple organ dysfunction syndromes (MODS) are diagnosed when two or more organs fail. (II) Continuous organ function damage: organ function damage lasts no less than 48 hours. (III) The diagnosis of acute renal injury is based on the network classification of acute renal injury (7). The diagnosis of acute respiratory distress syndrome was based on Berlin criteria (8). (IV) Continuous invasive arterial blood pressure monitoring was used to measure the changes of blood pressure at different time points, and the 24-hour systolic blood pressure variability (24h SBPV) and 24-hour diastolic blood pressure variability (24h DBPV) was calculated (9). 
Table 1 Comparison of general data between the two groups

\begin{tabular}{|c|c|c|c|c|c|c|}
\hline Group & $\mathrm{n}$ & Age (year) & Gender (male) & Body mass index $\left(\mathrm{kg} / \mathrm{m}^{2}\right)$ & Smoking history & Drinking history \\
\hline Control group & 102 & $42.51 \pm 13.46$ & 50 (49.02\%) & $23.69 \pm 2.81$ & $20(19.61 \%)$ & $15(14.71 \%)$ \\
\hline$t / \chi^{2}$ value & & 0.710 & 2.391 & 1.012 & 0.129 & 0.040 \\
\hline$P$ value & & 0.479 & 0.122 & 0.313 & 0.719 & 0.841 \\
\hline
\end{tabular}

Table 2 Comparison of $24 \mathrm{~h} \mathrm{SBPV}$ and $24 \mathrm{~h}$ DBPV between the two groups

\begin{tabular}{lccc}
\hline Group & $\mathrm{n}$ & 24h DBPV & 24h SBPV \\
\hline Observation group & 102 & $0.56 \pm 0.16$ & $0.56 \pm 0.16$ \\
Control group & 102 & $0.37 \pm 0.16$ & $0.36 \pm 0.17$ \\
$t$ value & & 8.836 & 8.889 \\
$P$ value & & 0.000 & 0.000 \\
\hline
\end{tabular}

24h SBPV, 24-hour systolic blood pressure variability; $24 \mathrm{~h}$ DBPV, 24-hour diastolic blood pressure variability.

\section{Statistical analysis}

The statistical analysis of this study was completed by SPSS 26.0. All tests were bilateral, and $\mathrm{P}<0.05$ was recognized that the difference was statistically significant. The measurement data conformed to normal distribution were presented as mean \pm standard deviation, and the measurement data accorded with non-normal distribution are expressed as mean (P25-P75). T-test was used for the measurement data conformed to normal distribution. Mann-Whitney $U$ test for two independent samples and Kruskal-Wallis H rank-sum test for multiple samples were used for the measurement data accorded with non-normal distribution. The rates of different categorical variables were compared by $\chi^{2}$ test and expressed as $n$ (percentage within the group).

\section{Results}

\section{Comparison of general data between the two groups}

There was no statistical significance in the comparison of general data, including age, gender, body mass index, smoking history, and drinking history between the two groups $(\mathrm{P}>0.05)$ (Table 1$)$.
Comparison of 24b SBPV and 24b DBPV between the two groups

Comparing with the control group, the $24 \mathrm{~h}$ DBPV in the observation group was significantly higher $(0.56 \pm 0.16$ vs. $0.37 \pm 0.16, \mathrm{P}=0.000)$, and the $24 \mathrm{~h} \mathrm{SBPV}$ increased significantly $(0.56 \pm 0.16$ vs. $0.36 \pm 0.17, \mathrm{P}=0.000)$ (Table 2).

\section{Correlation between 24b SBPV and clinical indicators in patients with sepsis}

Pearson linear correlation analysis showed that 24h SBPV was significantly positively correlated with the level of procalcitonin, APACHEII score, and SOFA score $(\mathrm{r}=0.301$, 0.216 and $0.218, \mathrm{P}<0.05$ ) (Table 3).

\section{Correlation between $24 b$ DBPV and clinical indexes in patients with sepsis}

24h DBPV was significantly positively correlated with the level of procalcitonin, APACHEII score, and SOFA score in patients with sepsis $(\mathrm{r}=0.302,0.212$ and $0.210, \mathrm{P}<0.05)($ Table 4$)$.

\section{Value of 24b SBPV and 24b DBPV in the diagnosis of multiple organ failure in patients with sepsis}

24h SBPV and 24h DBPV are of certain value in diagnosing multiple organ failure in patients with sepsis. The areas under the curve were [0.649 (95\% CI: 0.539-0.759), $\mathrm{P}=0.010]$ and $[0.650(0.540-0.760), \mathrm{P}=0.009]$, respectively (Table 5, Figure 1).

Value of 24b SBPV and 24b DBPV in the diagnosis of persistent organ failure in patients with sepsis

$24 \mathrm{~h} \mathrm{SBPV}$ and $24 \mathrm{~h} \mathrm{DBPV}$ are of certain value in the 
Table 3 Correlation between $24 \mathrm{~h}$ SBPV and clinical indicators in patients with sepsis

\begin{tabular}{lcccc}
\hline Category & Level of procalcitonin & Number of leukocytes & APACHEll score & SOFA score \\
\hline r value & 0.301 & -0.119 & 0.216 & 0.218 \\
$\mathrm{P}$ value & 0.002 & 0.235 & 0.029 & 0.028 \\
\hline
\end{tabular}

APACHEll score, Acute Physiology and Chronic Health Evaluation; SOFA score, sequential organ failure score; 24h SBPV, 24-hour systolic blood pressure variability.

Table 4 Correlation between 24h DBPV and clinical indexes in patients with sepsis

\begin{tabular}{lcccc}
\hline Category & Level of procalcitonin & Number of leukocytes & APPACHEll score & SOFA score \\
\hline$r$ value & 0.302 & -0.129 & 0.212 & 0.210 \\
$P$ value & 0.002 & 0.197 & 0.032 & 0.034 \\
\hline
\end{tabular}

APPACHEII score, Acute Physiology and Chronic Health Evaluation; SOFA score, sequential organ failure score; 24h DBPV, 24-hour diastolic blood pressure variability.

Table 5 Value of 24h SBPV and 24h DBPV in the diagnosis of multiple organ failure in patients with sepsis

\begin{tabular}{|c|c|c|c|c|}
\hline Category & Area under the curve & Standard deviation & $P$ value & $95 \% \mathrm{Cl}$ \\
\hline 24h DBPV & 0.650 & 0.056 & 0.009 & $0.540-0.760$ \\
\hline
\end{tabular}

24h SBPV, 24-hour systolic blood pressure variability; 24h DBPV, 24-hour diastolic blood pressure variability.

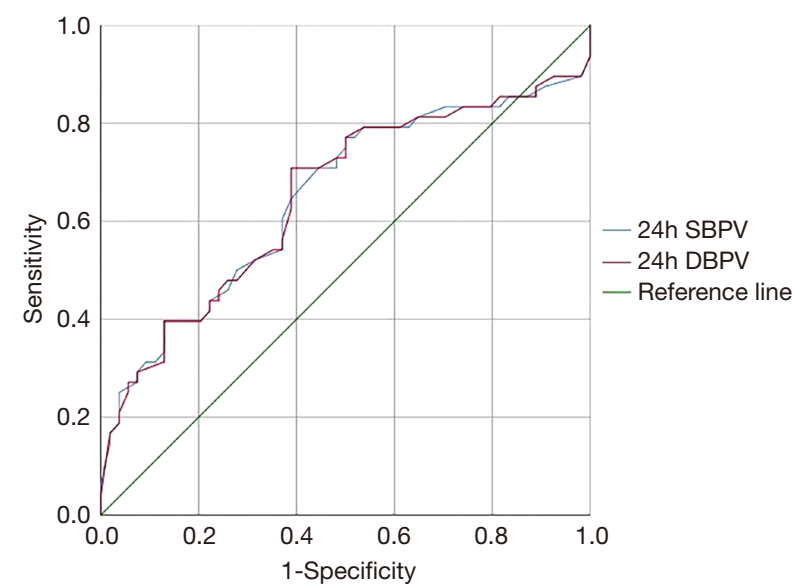

Figure 1 Value of $24 \mathrm{~h} \mathrm{SBPV}$ and $24 \mathrm{~h}$ DBPV in the diagnosis of multiple organ failure in patients with sepsis. 24h SBPV, 24-hour systolic blood pressure variability; 24h DBPV, 24-hour diastolic blood pressure variability.

diagnosis of persistent organ failure in patients with sepsis. The areas under the curve were $[0.647$ (95\% CI: $0.538-0.757), \mathrm{P}=0.010]$ and [0.647 (95\% CI: 0.538-0.757), $\mathrm{P}=0.010]$, respectively (Table 6, Figure 2).

\section{Predictive value of $24 b$ SBPV and $24 b$ DBPV on 28-day} mortality in patients with sepsis

24h SBPV and 24h DBPV have a certain value in predicting 28 -day mortality in patients with sepsis. The areas under the curve are $[0.668$ (95\% CI: $0.545-0.791$ ), $\mathrm{P}=0.016]$ and [0.667 (95\% CI: 0.544-0.790), $\mathrm{P}=0.017$, respectively (Table 7 and Figure 3).

\section{Discussion}

The 24-hour blood pressure rhythm of normal subjects showed a double peak to valley. Patients with hypertension have short-term BPV within one day. The variation of blood pressure indicates that the normal blood pressure rhythm disappears. Whether this variability decreases or increases, it will affect the self-regulation of the human body and cause damage to corresponding organs. In patients with a history of cardiovascular and cerebrovascular diseases, including hypertension, it has been confirmed that $\mathrm{BPV}$ is related to the injury of target organs (10-12). BPV is only a substitute index, and the underlying mechanism and factors that cause and affect its fluctuation are the essence. Organ dysfunction often accompanies sepsis patients, poor 
Table 6 Value of 24h SBPV and 24h DBPV in the diagnosis of persistent organ failure in patients with sepsis

\begin{tabular}{lcccc}
\hline Category & Area under the curve & Standard deviation & $\mathrm{P}$ value & $95 \% \mathrm{Cl}$ \\
\hline $24 \mathrm{~h}$ SBPV & 0.647 & 0.056 & 0.010 & $0.538-0.757$ \\
$24 \mathrm{~h}$ DBPV & 0.647 & 0.056 & 0.010 & $0.538-0.757$ \\
\hline
\end{tabular}

24h SBPV, 24-hour systolic blood pressure variability; 24h DBPV, 24-hour diastolic blood pressure variability.

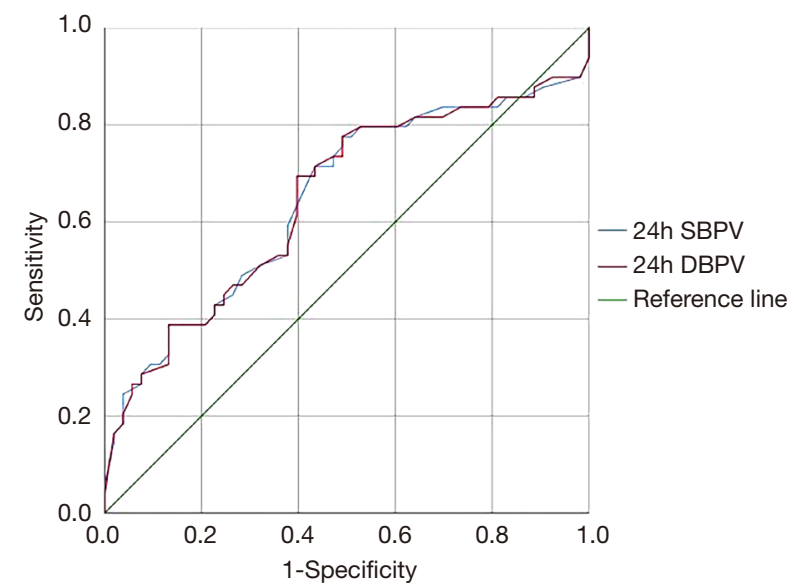

Figure 2 Value of $24 \mathrm{~h} \mathrm{SBPV}$ and $24 \mathrm{~h}$ DBPV in the diagnosis of persistent organ failure in patients with sepsis. 24h SBPV, 24-hour systolic blood pressure variability; 24h DBPV, 24-hour diastolic blood pressure variability.

tissue perfusion, or hypotension. Therefore, there may also be the disappearance of blood pressure rhythm, which is manifested as the change of BPV. However, relevant studies focus mainly on the role of BPV in patients with cardiovascular and cerebrovascular diseases, and there are few studies on BPV in patients with sepsis.

The increase of BPV indicates that the patient's normal blood pressure rhythm disappears. The disappearance of this normal blood pressure rhythm may be due to insufficient blood volume, inflammatory reaction, immune dysfunction, hypermetabolism, and abnormal autonomic nervous function after sepsis. In the early stages of patients with septic shock, the blood pressure can remain normal or decrease due to the compensatory effect. The blood pressure decreases significantly entering the decompensation period. During this period, the mean arterial pressure decreases, and the blood pressure fluctuates greatly, so it can be manifested as the increase of BPV $(\mathrm{BPV}=$ standard deviation of blood pressure at different time points over a period of time/mean blood pressure in the same period). In addition, the autonomic nervous function also has a significant impact on BPV. When the autonomic nervous function is disordered, the body's ability to control blood pressure rhythm decreases, leading to apparent fluctuations in blood pressure and increases BPV. In 2014, Pandey's study showed that BPV was elevated in patients with sepsis and had a certain value in predicting mortality (13). Two other related studies also confirm it $(4,5)$ supporting our study. The results of our study show that the $24 \mathrm{~h} \mathrm{SBPV}$ and $24 \mathrm{~h}$ DBPV in patients with sepsis are significantly increased, and positively correlated with the level of procalcitonin, APACHEII score, and SOFA score. It has a certain value in predicting multiple organ failure, persistent organ failure, and 28-day mortality. Our study has a certain value compared with previous studies, mainly reflected in the following aspects: (I) the number of cases in this study was more than that in other studies; (II) there was a control group in this study; (III) this study focused on the target organ function of patients, while previous studies only paid attention to the correlation between BPV and mortality. In addition, the level of procalcitonin, APACHEII score, and SOFA score are important indicators representing the severity of sepsis (14-16). The higher the level of these indicators, the more serious the sepsis. Multiple organ failure and persistent organ failure are among the most important risk factors for poor prognosis (17-19), and 28-day mortality is the most important prognosis index. Combined with the results of this study, BPV has a substantial value in predicting the prognosis of patients with sepsis, which is closely related to the severity of sepsis, and it is worthy of further study. Moreover, the monitoring time of BPV and DBPV should be further extended. Finally, we believe that procalcitonin, APACHEII score and SOFA score combined with BPV can judge the severity of sepsis more accurately.

The primary deficiency of this study is that this study is a retrospective clinical study, and the number of cases is relatively insufficient. Secondly, assessing BPV also needs many other indicators. Limited to the fact that this study is a retrospective clinical study, other indicators of BPV, such as resting blood pressure variability, cannot be obtained. 
Table 7 Predictive value of 24h SBPV and 24h DBPV on 28-day mortality in patients with sepsis

\begin{tabular}{|c|c|c|c|c|}
\hline Category & Area under the curve & Standard deviation & $P$ value & $95 \% \mathrm{Cl}$ \\
\hline 24h DBPV & 0.667 & 0.063 & 0.017 & $0.544-0.790$ \\
\hline
\end{tabular}

24h SBPV, 24-hour systolic blood pressure variability; 24h DBPV, 24-hour diastolic blood pressure variability.

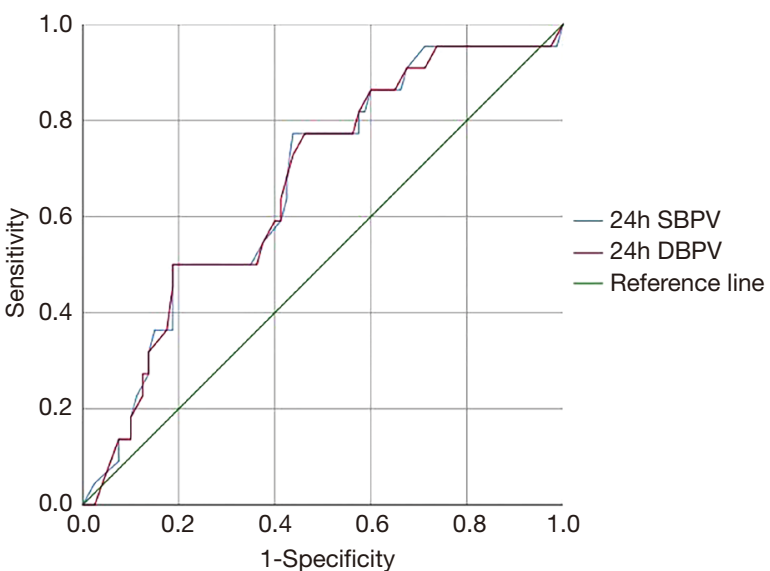

Figure 3 Predictive value of $24 \mathrm{~h} \mathrm{SBPV}$ and $24 \mathrm{~h}$ DBPV on 28-day mortality in patients with sepsis. 24h SBPV, 24-hour systolic blood pressure variability; 24h DBPV, 24-hour diastolic blood pressure variability.

The increased BPV in patients with sepsis is of certain value in predicting the prognosis and organ function injury in patients with sepsis.

\section{Acknowledgments}

Funding: None.

\section{Footnote}

Reporting Checklist: The authors have completed the STARD reporting checklist. Available at https://dx.doi. org/10.21037/apm-21-2183

Data Sharing Statement: Available at https://dx.doi. org/10.21037/apm-21-2183

Conflicts of Interest: All authors have completed the ICMJE uniform disclosure form (available at https://dx.doi. org/10.21037/apm-21-2183). The authors have no conflicts of interest to declare.
Ethical Statement: The authors are accountable for all aspects of the work in ensuring that questions related to the accuracy or integrity of any part of the work are appropriately investigated and resolved. This study is a retrospective clinical study that can do without patient informed consent. The protocol of this study is in line with the Declaration of Helsinki (as revised in 2013). It retrospectively analyzes the imaging and clinical data left by patients, and does not use serum or tissue for additional tests. It is an observational test that can do without the approval of the ethics committee.

Open Access Statement: This is an Open Access article distributed in accordance with the Creative Commons Attribution-NonCommercial-NoDerivs 4.0 International License (CC BY-NC-ND 4.0), which permits the noncommercial replication and distribution of the article with the strict proviso that no changes or edits are made and the original work is properly cited (including links to both the formal publication through the relevant DOI and the license). See: https://creativecommons.org/licenses/by-nc-nd/4.0/.

\section{References}

1. Triantafyllidi H, Benas D, Schoinas A, et al. Hypertensionmediated organ damage regression associates with blood pressure variability improvement three years after successful treatment initiation in essential hypertension. J Clin Hypertens (Greenwich) 2021;23:1150-8.

2. Cuspidi C, Carugo S, Tadic M. Blood pressure variability and target organ damage regression in hypertension. J Clin Hypertens (Greenwich) 2021;23:1159-61.

3. Barochiner J, Martínez R, Aparicio LS. Novel Indices of Home Blood Pressure Variability and HypertensionMediated Organ Damage in Treated Hypertensive Patients. High Blood Press Cardiovasc Prev 2021;28:365-72.

4. Tang Y, Sorenson J, Lanspa M, et al. Systolic blood pressure variability in patients with early severe sepsis or septic shock: a prospective cohort study. BMC Anesthesiol 
2017;17:82.

5. Nouriel JE, Millis SR, Ottolini J, et al. Blood pressure variability as an indicator of sepsis severity in adult emergency department patients. Am J Emerg Med 2018;36:560-6.

6. Singer M, Deutschman CS, Seymour CW, et al. The Third International Consensus Definitions for Sepsis and Septic Shock (Sepsis-3). JAMA 2016;315:801-10.

7. Mehta RL, Kellum JA, Shah SV, et al. Acute Kidney Injury Network: report of an initiative to improve outcomes in acute kidney injury. Crit Care 2007;11:R31.

8. ARDS Definition Task Force; Ranieri VM, Rubenfeld GD, et al. Acute respiratory distress syndrome: the Berlin Definition. JAMA 2012;307:2526-33.

9. Tran QK, Najafali D, Tiffany L, et al. Effect of Blood Pressure Variability on Outcomes in Emergency Patients with Intracranial Hemorrhage. West J Emerg Med 2021;22:177-85.

10. Lin JY, Kuo KL, Kuo YH, et al. Association between real-world home blood pressure measurement patterns and blood pressure variability among older individuals with hypertension: A community-based blood pressure variability study. J Clin Hypertens (Greenwich) 2021;23:628-37.

11. Nam JH, Park JI, Kim BJ, et al. Clinical impact of blood pressure variability in patients with COVID-19 and hypertension. Blood Press Monit 2021;26:348-56.

12. Visaria A, Raju P, Islam S, et al. Understanding the impact of blood pressure guidelines and variability on

Cite this article as: Zhu Y, Li Y, Cai W, Zhang C. Predictive value of blood pressure variability for organ dysfunction in patients with sepsis. Ann Palliat Med 2021;10(9):9678-9684. doi: 10.21037/apm-21-2183 hypertension diagnoses. J Hypertens 2021;39:1044-5.

13. Pandey NR, Bian YY, Shou ST. Significance of blood pressure variability in patients with sepsis. World J Emerg Med 2014;5:42-7.

14. Song J, Moon S, Park DW, et al. Biomarker combination and SOFA score for the prediction of mortality in sepsis and septic shock: A prospective observational study according to the Sepsis-3 definitions. Medicine (Baltimore) 2020;99:e20495.

15. Patil NB, Jotkar SK. A Comparative study of quickSOFA score and serum lactate levels with SOFA score for predicting the clinical outcome in patients with sepsis. J Assoc Physicians India 2020;68:86.

16. Tambo M, Taguchi S, Nakamura Y, et al. Presepsin and procalcitonin as predictors of sepsis based on the new Sepsis-3 definitions in obstructive acute pyelonephritis. BMC Urol 2020;20:23.

17. Bline KE, Moore-Clingenpeel M, Hensley J, et al. Hydrocortisone treatment is associated with a longer duration of MODS in pediatric patients with severe sepsis and immunoparalysis. Crit Care 2020;24:545.

18. Im Y, Yoo H, Lee JY, et al. Association of plasma exosomes with severity of organ failure and mortality in patients with sepsis. J Cell Mol Med 2020;24:9439-45.

19. Kim H, Hur M, Struck J, et al. Proenkephalin Predicts Organ Failure, Renal Replacement Therapy, and Mortality in Patients With Sepsis. Ann Lab Med 2020;40:466-73.

(English Language Editor: J. Chapnick) 